Physics of Plasmas, to appear, 2002.

\title{
Radiation sources and diagnostics with ultrashort electron bunches
}

\author{
P. Catravas, E. Esarey, W.P. Leemans \\ l'OASIS Group, Center for Beam Physics, Lawrence Berkeley National Laboratory, University of \\ California,Berkeley, CA 94720
}

(November 2, 2001)

\begin{abstract}
The basic principles and design of radiation sources (transition radiation, Cerenkov radiation, radiation from periodic structures, etc) and radiationbased diagnostics will be discussed, with emphasis on radiation from ultrashort electron bunches. Ultrashort electron bunches have the potential to produce high peak flux radiation sources that cover wavelength regimes where sources are currently not widely available (coherent $\mathrm{THz} / \mathrm{IR}$ ) as well as ultrashort X-ray pulses (3-100 fs). While radiation from the electron bunch contains the full signature of the electron beam and/or medium it has travelled through, the deconvolution of a single property of interest can be difficult due to a large number of contributing properties. The experimental implementation of novel solutions to this problem will be described for beams from $30 \mathrm{MeV}$ to $30 \mathrm{GeV}$, including fluctuational interferometry, source imaging, phase matched cone angles and laser-based techniques, which utilize optical transition radiation, wiggler and Cerenkov radiation, and Thomson scattering. These novel diagnostic methods have the potential to resolve fs bunch durations, slice emittance on fs scales, etc. The advantages and novel features of these techniques will be discussed.
\end{abstract}




\section{INTRODUCTION}

Interest in the production of ultrashort electron bunches has been rapidly growing, motivated by possible applications in ultrafast science. For example, the typical timescale for lattice changes in a crystal is related to the characteristic vibrational time, $\sim 100 \mathrm{fs}$. Such bunches are currently under development using various production mechanisms, including laser-driven accelerators [1] and inverse free electron lasers [2]. Laser wakefield accelerators [3], which are implemented with ultrafast chirped pulse amplification (CPA) laser systems [4], offer intriguing potential for producing ultrashort radiation pulses from X-rays to $\mathrm{THz}$ frequencies in synchronism with the high power, ultrafast laser, making all-optical electron injectors of interest for pump-probe experiments. The diagnostic techniques presented here are well suited for ultrashort electron bunches and can provide an essential tool in the development and control of advanced accelerators.

It has long been recognized that radiation from electron beams contains the full signature of the beam parameters, including divergence, spot size, energy, energy spread, pointing and bunch shape, while offering numerous appealing features [5-8]. The techniques are generally non-destructive, allowing further use of the beam downstream on the same shot. Often, radiation provides the means to measure parameters precisely at interaction region of an experiment. The radiation is prompt and thus permits time-resolved measurements of beam parameters to be implemented. Finally, linearity can be superior to other methods, where signal saturation at high charge is a common problem (e.g., for phosphor screens). The difficulty with radiation-based diagnosis lies with the fact that the signatures of each critical beam parameter in the radiation pattern or spectrum are often similar, so that deconvolution of a single parameter is difficult. Here examples are described from recent diagnostic experiments illustrating how the challenge of distilling a single parameter of interest can be met for a variety of parameters over a wide range of beam energies. These techniques include fluctuational interferometry using incoherent radiation for ultrashort bunch diagnosis, source imaging and interferometric techniques, the use of phase-matched cone angles, and 
laser-based methods which enable microprobing of fs slices of the electron beam.

The utility of these techniques has been demonstrated in a number of recent experiments [7-26]. Fluctuational interferometry has been utilized to diagnose picosecond bunches using a simple and versatile experimental setup, using spikes of width $1 / \tau_{b}$ in incoherent emission spectra, a feature easier to resolve as the bunchlength decreases to fs lengths [9-12]. Experiments addressing practical issues which arise at ultrarelativistic energies are presented, including first measurements performing single shot transverse profiling of $50 \mu \mathrm{m}$ spots at $30 \mathrm{GeV}$, which illustrate the special utility of transition radiation in the visible wavelengths for electron beams of energy $30 \mathrm{GeV}$ and higher [20]. These techniques have been applied to a plasma-based wakefield acceleration experiment at $30 \mathrm{GeV}$ [21], to study transverse focussing dynamics and the behavior of tails in the electron beam. The use of prompt radiation from the electron beam is illustrated with Cerenkov radiation at $30 \mathrm{GeV}$, with which the properties of a partially ionized medium have been diagnosed with automatic time and trajectory synchronism with the beam [22]. A comprehensive family of diagnostic techniques based on the phase matched cone of the wiggler radiation spatial profile is presented which provide excellent sensitivity to beam energy, and the means to separate and diagnose low divergences and small changes in energy spread [8,23]. Slice measurements $(>1$ ps resolution) were performed. Ultrafast laser-based techniques offer the possibility of measuring the properties of femtosecond slices of an electron bunch, which has been demonstrated in recent experiments. A family of fs slice diagnostic techniques has been demonstrated with Thomson scattering [24-26].

\section{FLUCTUATIONAL INTERFEROMETRY}

Noise has long been used to infer properties of physical systems [27]. A versatile technique for non-perturbative diagnosis of electron beam bunchlength via shot-noise driven fluctuations in incoherent radiation was recently proposed $[9,10]$. Any source of incoherent emissions may be used (i.e. wiggler, synchrotron, transition radiation, Cerenkov radation, 
Smith Purcell, etc.), provided that the number of photons is sufficiently high to neglect quantum effects. The technique is appropriate for ultrashort bunches, and can be implemented with a straightforward experimental setup for bunchlengths which are at or beyond the current state-of-the-art streak camera resolution (well below 1 ps). A proof of principle experiment of this technique has been performed $[8,11]$

The electric field of a collection of charged particles can be written in the frequency domain as

$$
E(\omega)=e(\omega) \sum_{j} e^{i \omega t_{j}}
$$

where $\omega$ is the radiation frequency, $t_{j}$ is the time of arrival of the jth electron and $|e(\omega)|^{2}$ is the single electron spectrum. Incoherent radiation will occur when $\omega \tau_{b}>>1$. Shot noise, which follows Poisson statistics, appears in the incoherent spectrum as spikes of random amplitude and central frequency having a characteristic width $1 / \tau_{b}$. If the collected radiation is limited to a single transverse mode (i.e. is transversely coherent) and the spectral instrumental resolution is much better than $1 / \tau_{b}$, then $100 \%$ modulation of spectral intensity, which arises from the random contributions of individual phasors in Eqn. 1, can be fully resolved. In this case, longitudinal phase space information can be extracted directly from the measured spike width. When the spectral resolution is such that $1 / \tau_{\text {inst }}>>1 / \tau_{b}$, where $\tau_{\text {inst }} \sim 1 / d \omega$ is the coherence time associated with a frequency measurement bin of width $d \omega$, then the individual frequency measurement bin will contain $p=\left(1 / \tau_{\text {inst }}\right) /\left(1 / \tau_{b}\right)$ independent spikes (i.e. $p$ longitudinal modes). Each frequency measurement bin in the single shot spectrum will be uncorrelated and fluctuate with a variance, $\sigma$, equal to $1 / \sqrt{p}$. By performing the measurement in the frequency domain, the variance can be obtained in a single shot from the statistics of uncorrelated frequency bins, and the bunchlength can be extracted from $\tau_{b}=\tau_{\text {inst }} / \sigma^{2}$.

Transverse phase space information can be introduced into the spectral fluctuations by collecting more than one transverse mode, $q$. When the radiation is not transversely coherent, the level of modulation of the spectrum will decrease from 100\%, as if $q$ independent 
spectra were averaged in the detector. The spectral intensity distribution, $f(x, \kappa)$, and correlation of spectral intensity provide functional tools for quantifying the number of longitudinal and transverse modes in a measured spectrum, allowing longitudinal and transverse phase space information to be extracted. The intensity distribution for $\kappa$ independent Poisson processes is given by the gamma distribution,

$$
f(x, \kappa)=\frac{x^{\kappa-1} \kappa^{\kappa}}{\Gamma(\kappa)} e^{-\kappa x}
$$

where $\mathrm{x}$ is normalized intensity, $x=I /<I>$ and $\kappa$ is the product of the number of longitudinal and transverse modes, $\kappa=p q$. In the limit of one independent process, $\kappa=1$ (i.e. spectral resolution exceeding the characteristic spike width $-1 / \tau_{\text {inst }}<<1 / \tau_{b}$ and a zero emittance electron beam) Eqn. 2 reduces to the exponential distribution, and the fluctuational characteristics depend only on the bunchlength.

The correlation of spectral intensity, $C_{\text {meas }}$, is defined as

$$
C_{\text {meas }}(n)=<I\left(\omega_{i}\right) I\left(\omega_{i+n}\right)>/<I\left(\omega_{i}\right)^{2}>
$$

where $I\left(\omega_{i}\right)$ is the spectral intensity in the ith frequency bin and $n$ is the shift in frequency bin. An analytic expression for the correlation of spectral intensity can be written as a function of the product $\kappa=p q$ and the fourier transform of the current distribution, $\rho(\omega)$ $[11]$

$$
C_{f i t}(n)=(1-a) \frac{\left[\rho\left(\omega_{n}\right)^{2} * S\left(\omega_{n}\right)\right]}{\left[\rho\left(\omega_{0}\right)^{2} * S\left(\omega_{0}\right)\right]}+a
$$

where

$$
a=\frac{1}{1+1 / \kappa}
$$

$S(\omega)$ is the instrumental impulse response and $\omega_{0}$ and $S_{0}$ are evaluated at zero frequency shift $(\mathrm{n}=0)$. The number of longitudinal modes, which depends on the bunchlength, determines the behavior of $C_{\text {meas }}$ at small shift, $n$, while the asymptote at large $\mathrm{n}$ depends on the product $p q$. Thus, it is possible to separate and extract both bunchlength and emittance. 
The experimental setup is shown in Fig. 1. Measurements were performed at the Accelerator Test Facility (ATF) [28] at Brookhaven National Laboratory (BNL), which provided $44 \mathrm{MeV}$ electron bunches variable from 1-5 ps, with corresponding charge 100-400 pC, a few $\pi$ mm.mrad normalized emittance and $0.7 \%$ full width energy spread. Incoherent radiation in the visible wavelengths $(622 \mathrm{~nm})$ was produced using the MIT microwiggler [29], which provided a $0.42 \mathrm{~T}$ peak on-axis field over 60 periods of $8.8 \mathrm{~mm}$ each. The emissions were collected and transported to a $0.27 \mathrm{~m}$ focal length spectrometer with a 1200 groove per $\mathrm{mm}$ visible grating (SPEX model 270M). The single shot spectrum was recorded with a Gen IV image intensifier having 35-40\% quantum efficiency. The spectral resolution was measured using a He-Ne laser and was intensifier-limited to $0.07 \mathrm{~nm}$.

Experimental measurements of single shot spectra for electron bunchlengths of 1.5 and 4.5 ps are shown in Fig. 2, along with spectra generated numerically using Eqn. 1 for the experimental conditions. The intensity distribution and correlation of spectral intensity for these two bunchlengths are shown in Fig. 3, and indicate that the data is well described by the gamma distribution. For wiggler radiation, with a collection angle fixed at the single electron radiation opening angle, $1 /\left(\sqrt{N_{w}} \gamma\right)$, and a matched electron beam, the product of the number of transverse and longitudinal modes is given by [11]

$$
\kappa=p q=\frac{1 / \tau_{\text {inst }}}{1 / \tau_{b}}\left[1+\frac{\epsilon_{b}}{\lambda / 4 \pi}\right]
$$

where $N_{w}$ is the number of wiggler periods, $\epsilon_{b}$ is the beam emittance, and $\lambda$ is the radiation wavelength. The values of $k$ extracted from Fig. 3 were 2.0 and 3.6, and corresponded to bunchlengths of 2 and $5.5 \mathrm{ps}$ and emittances of 1.5 and $5.5 \pi \mathrm{mm} \mathrm{mrad}$, in agreement with independent measurements provided by the ATF. While these estimates included a form factor for bunch shape, the full bunch shape can also be extracted directly from fluctuational interferometry [9], [12]. 


\section{SOURCE IMAGING AND INTERFEROMETRIC TECHNIQUES}

Since its introduction in the first half of the last century by Frank and Ginzburg [30], transition radiation (TR) has been widely used in high energy physics for particle identification and has proven an essential tool (see section V) in beam diagnosis from 10's to 100's of $\mathrm{MeV}$. Until very recently, however, most applications of TR at ultrarelativistic energies have been in the X-ray wavelengths [31], while the visible wavelength range has been underutilized at energies beyond a few GeV. Spot size resolution is determined by the diffraction limited spot size, $d=\lambda / 2 \pi \theta_{\text {coll }}$, where $\lambda$ is the observation wavelength and $\theta$ is the collection angle. Most radiation sources (e.g. synchrotron radiation) are primarily contained within a cone width of $1 / \gamma,(\gamma$ is the relativistic factor of the electron beam) making it difficult to obtain the resolution for transverse beam sizes of 10's of microns using visible wavelengths at large $\gamma$ without integrating a very large number of shots. At $30 \mathrm{GeV}\left(\gamma=6 \times 10^{4}\right)$ and $\lambda=632$ $\mathrm{nm}, d \sim 1 \mathrm{~cm}$. However, while the TR spatial profile peaks at $1 / \gamma$, the intensity drops off slowly outside the $1 / \gamma$ peak, providing the key to spot size and divergence resolution.

Recently, it was demonstrated experimentally that the full transverse profile of 50 micron electron beams can be obtained in a single shot and $100 \mu \mathrm{rad}$ divergences obtained in 5 shots using visible TR at $30 \mathrm{GeV}$, the first such measurements at this beam energy [20]. Moreover, because angles much greater than $1 / \gamma$ were utilized, spatial and angular resolution become independent of beam energy, and these results can be extended to machines with energies higher than $30 \mathrm{GeV}$.

The TR angular distribution from a single electron for a metal foil is [13]

$$
\frac{d^{2} W_{1}(\theta)}{d \omega d \Omega} \sim \frac{\theta^{2}}{\left(\gamma^{-2}+\theta^{2}\right)^{2}} \approx \frac{1}{\theta^{2}}, \frac{1}{\gamma} \ll \theta \ll 1
$$

where $\frac{d^{2} W_{1}(\theta)}{d \omega d \Omega}$ is the energy radiated per unit frequency $d \omega$ and solid angle $\delta \Omega$, and $\theta$ is the angle with respect to the electron trajectory. While both spot size and divergence can be obtained from single foil TR, divergence measurements often utilize the Wartski interferometer [13](Fig. 4) to enhance sensitivity to beam divergence. Forward radiation 
from the upstream foil interferes with backward radiation from the downstream foil, yielding an addition of phases, $1-\exp \left[-j \pi\left(\gamma^{-2}+\theta^{2}\right) L / \lambda\right]$, where the interference term depends on the formation length, $L_{f}=(\lambda / \pi)\left(\gamma^{-2}+\theta^{2}\right)^{-1}$ (assuming vacuum, $\gamma^{2}>>1$ and $\theta^{2}<<1$. The angular intensity distribution for the 2-foil interferometer is given by [13],

$$
\frac{d^{2} W_{2}(\theta)}{d \omega d \Omega} \sim \frac{1}{\theta^{2}} \sin ^{2}\left(\frac{L}{2 L_{f}}\right)
$$

where $L_{f} \simeq \lambda /\left(\pi \theta^{2}\right)$. Implementations of the Wartski interferometer often use a foil separation, $L$, such that the first interference maximum is set near $1 / \gamma$. For experiments from $20 \mathrm{MeV}-100 \mathrm{MeV}$, typical L's range from < $1 \mathrm{~mm}$ to slightly over a centimeter [13], $[14,15,17,32,18,24,16]$. At $30 \mathrm{GeV}$, the formation length at $1 / \gamma$ is $\lambda \gamma^{2} / 2 \pi$ and a similar design thus requires an unreasonable foil separation of $1 \mathrm{~km}$. Once again, the slow drop-off of the intensity distribution at large angles provides the key, as it allows the interference pattern to be confined to the wings, enabling smaller foil separations. Divergence resolution is given by $\sigma_{r e s}=d / L$, and $d$ can be made small by increasing the collection angle while maintaining a reasonable foil separation, $L$.

TR experiments [20] were performed at the Stanford Linear Accelerator Center (SLAC) Final Focus Test Beam (FFTB), with a $28.5 \mathrm{GeV}$ electron beam, and single microbunches of $1.5 \times 10^{10}$ particles with normalized vertical and horizontal emittances of $0.47 \times 10^{-5} \mathrm{~m}-\mathrm{rad}$ and $6.5 \times 10^{-5} \mathrm{~m}-\mathrm{rad}$ (Fig. 4). An aluminum coated fused silica substrate $(150 \mu \mathrm{m}$ thick) was located $0.56 \mathrm{~m}$ downstream from a retractable, $25 \mu \mathrm{m}$ thick Beryllium foil. Imaging resolution of the beam at the foil location $\left(\theta_{\text {coll }} \sim 0.1 \mathrm{rad}\right)$ was $<10 \mu \mathrm{m}$, while $6 / \gamma$ radians per pixel were resolved in radiation angular distribution measurements. Fig. 5a shows images of the single shot transverse profiles and rms dependence obtained with optical transition radiation (OTR) for a quadrupole scan, with minimum spot sizes of 50 microns in agreement with an independent measurement performed with a wire scanner. Measurements of the angular intensity distribution of the two-foil interferometer are shown for low and high beam divergence. The upper image shows clean modulation in the vertical, and loss of modulation in the horizontal, while the lower image is cleanly modulated in both axes. This 
is consistent with the known difference in vertical and horizantal emittance at the FFTB. The divergence extracted from Fig. $5 \mathrm{~b}$ was $\sigma_{\theta x} 100 \mu \mathrm{rad}$.

This diagnostic was developed in support of the E157 experiment [21], which studied the interaction of a $30 \mathrm{GeV}$ beam with a long plasma column (see below), and was used to study the transverse dynamics of the electron beam in the plasma (Fig. 8b), to quantify the focussing strength of the plasma and to monitor tails on the electron beam.

\section{PHASE-MATCHED CONE ANGLE TECHNIQUES}

\section{A. Diagnosis of beam-plasma experiments with Cerenkov radiation}

Cerenkov radiation has been widely applied in high energy physics for particle identification, through use of the Cerenkov cutoff condition, $\gamma^{-2}+\theta_{c}^{2} \sim 2(n-1)$ (small angles), which depends on the electron velocity, $v$, where $\gamma=\left(1-v^{2} / c^{2}\right)^{-1 / 2}, n$ is the index of refraction of the medium, and $\theta_{c}$ is the CR cone angle. In contrast to the usual implementations, recent experiments operated in the limit $\gamma^{-2}<<n-1$, at $30 \mathrm{GeV}$ and $1 / \gamma^{2} \sim 10^{-10}$, which allows measurement of the properties of the medium, rather than the e-beam [22]. CR [33] is produced when the speed of light in the medium, $c / n$, is less than that of the electrons. The index of refraction of the medium, neglecting absorption, can be modelled by [34]

$$
n-1 \sim \sum_{\text {species }, s} \frac{N_{s} e^{2} f_{i k}}{2 m_{e} \epsilon_{0}\left(\omega_{r e s}^{2}-\omega^{2}\right)}-\frac{N_{e}}{2 N_{c r}}
$$

where $N_{s}$ is the species density (neutrals and/or ions), $f_{i k}$ is the oscillator strength, $\omega_{\text {res }}$ is the frequency of the atomic resonance, $N_{e}$ is the electron plasma density, and $N_{c r}=\omega^{2} m_{e} \epsilon_{0} / e^{2}$ is the critical density at the observation frequency $\omega$. Operation on the long wavelength side of an atomic spectral resonance results in Cerenkov radiation with an intensity distribution that is peaked at the phase matched cone angle, $\theta_{c}^{2} \sim 2(n-1)$ (small angles), and can

provide a measure of changes in $N_{s}$ (e.g. due to ionization), $N_{e}$ and $\omega_{\text {res }}$ on the timescale of the e-beam bunchlength. 
Plasma density causes inward shifting of the CR cone. The fractional ionization, $\left(N_{i} / N_{n}\right)$, where $N_{i}$ is the ion density, can be quantified from $\theta_{c}$ when the observation wavelength is chosen sufficiently far from resonance so that shifts in $\omega_{\text {res }}$ can be neglected. A baseline measurement without plasma gives the initial neutral density. Depletion of the neutral population reduces the cone angle and the ratio to the baseline angle will be proportional to $\sqrt{1-N_{i} / N_{n}}$.

In the E157 plasma wakefield acceleration experiment [21] at the SLAC FFTB, a 2 ps (rms) long, $30 \mathrm{GeV}$ e-beam bunch with $2 \times 10^{10}$ particles in a 30-100 $\mu \mathrm{m}$ spot traverses a $\mathrm{Li}$ heat pipe oven [35] (Fig. 6). In this scheme, the electron bunch blows out plasma electrons, producing transverse focussing forces along the bunch (that lead to betatron oscillations in the e-beam envelope) and longitudinal accelerating forces as the plasma electrons return at the electron bunch tail. Precise control over the value of plasma density is required to optimize both the longitudinal field and the fraction of the tail electrons which can experience acceleration.

Measurements described below were taken with a Li heat pipe oven of length $1.35 \mathrm{~m}$ plugged by $\mathrm{He}$ at a partial pressure of 450 mTorr. The Li neutral density was typically $\sim 10^{15} \mathrm{~cm}^{-3}$. An ArF Excimer laser operating at $193 \mathrm{~nm}$ (UV) partially ionized the Li. The UV was incoupled and outcoupled by dielectric coated fused Silica foils, $150 \mu \mathrm{m}$ thick, separated by $2.5 \mathrm{~m}$. The opposite side of the foil was coated with $\mathrm{Al}$ for e-beam spot size monitoring using OTR [20]. Images of the intensity distribution at the downstream foil and radiation angular distribution upstream and downstream were measured with 14 and 16 bit cooled CCD's. The plasma density seen by the e-beam was scanned by varying the delay between ionizing laser pulse and e-beam.

The Cerenkov cone response was benchmarked by measuring the dependence of the CR cone angle $\theta_{c}$ on oven temperature, $\mathrm{T}$, and pressure. The neutral density scaling with $\mathrm{T}$ as the Li vapor changes state is $N_{n}=N_{o}\left(T_{o} / T\right) \exp \left(-r_{o} / R T\right)$, where $r_{o}$ is the heat of vaporization, $\mathrm{R}$ is the universal gas constant, and $N_{o}$ is the density at the temperature $T_{o}$ [22]. $N_{n}$ estimated from $\theta_{c}$ vs $\mathrm{T}$ is plotted in Fig. 7 along with known values [36]. The heat 
of vaporization, $r_{o}$, determined from the slope of Fig. 7 came within $5-10 \%$ of the known value for $\mathrm{Li}, 148.13 \mathrm{~kJ} / \mathrm{mol}$ both at 676 and $700 \mathrm{~nm}$.

Plasma density extracted from the CR cone radius is shown in Fig. 8a for a scan of electron beam delay with respect to the time of fire of the ionizing laser. The estimated initial plasma density is $2.6 \times 10^{14} \mathrm{~cm}^{-3}$. The plasma density extracted from the CR cone can be compared with that required to produce the observed number of betatron oscillations extracted from the OTR images of the e-beam transverse profile simultaneously recorded in the images of the downstream foil (see center of the images in Fig. 7). The relation between plasma density and the betatron wavelength, $\lambda_{\beta}$, assuming that the plasma ions exert a linear restoring force on the e-beam, is given by $\lambda_{\beta}=\sqrt{2 \gamma} \lambda_{p} \propto N_{e}^{-1 / 2}$ [21]. Plasma density diagnosis based on measurement of betatron oscillations has been studied experimentally [37]. Plasma density extracted from the Cerenkov cone radius came within a factor of two of that extracted using the betatron oscillation technique (Fig. 8b).

\section{B. Single shot techniques with wiggler phase matched cone angle}

Phase matched cones in wiggler radiation spatial profiles can also provide beam parameters. While beam diagnosis with wiggler radiation has been pursued both theoretically and experimentally [6], these techniques typically make use of the on-axis radiation. The opening angle of the on-axis wiggler radiation cone for a wiggler with $N_{w}$ periods, $1 / \sqrt{N_{w}} \gamma$, limits the resolution for certain parameters, such as beam divergence, and has commonly forced diagnosis to be performed with harmonics rather than the fundamental. The separation of different inhomogeneous broadening contributions has been also been a troublesome issue.

Recent experiments have demonstrated single shot techniques for individually measuring an array of electron beam parameters using visible emissions at the fundamental, including a measurement of divergence much smaller than $1 / \sqrt{N_{w}} \gamma[8]$. The spatial profile of wiggler emissions into a Cerenkov cone selected by a narrow bandwidth interference filter is given by the phase matching condition [5], $\lambda=\left(\lambda_{w} / 2 \gamma^{2}\right)\left(1+a_{w}^{2} / 2+\gamma^{2} \theta_{\text {cone }}^{2}\right)$, where $\lambda$ is the 
observation wavelength, $\lambda_{w}$ is the wiggler period, $a_{w}$ is the wiggler parameter (normalized wiggler magnetic field amplitude), and $\theta_{\text {cone }}$ is the cone angle. The radius of the cone can be controlled by varying the beam energy or filter central wavelength, while the cone width depends on the number of wiggler periods, $N_{w}$, and is further broadened by the beam energy spread, the beam divergence and the filter bandwidth.

At a fixed frequency, the scaling of cone width for increasing cone angle is different for the various broadening mechanisms, permitting them to be separately diagnosed. The cone width $\sigma_{\text {cone }}$ in the wiggle plane consists of contributions from the number of wiggler periods, $\left(\sigma_{\text {cone, } N_{w}}\right)$, energy spread $\sigma_{c o n e, \gamma}$ and divergence $\sigma_{\text {cone, } x^{\prime}}[8]$ :

$$
\begin{gathered}
\sigma_{\text {cone }, N_{w}}=\frac{1}{4 N_{w}} \frac{1+a_{w}{ }^{2} / 2}{\gamma^{2} \theta_{\text {cone }}}, \theta_{\text {cone }}>>\frac{1}{\sqrt{N_{w}} \gamma}, N_{w}>>1, \\
\sigma_{\text {cone }, \gamma}=\frac{\sigma_{\gamma}}{\gamma} \frac{1+a_{w}{ }^{2} / 2}{\gamma^{2} \theta_{\text {cone }}} \\
\sigma_{\text {cone }, x^{\prime}}=\sigma_{x^{\prime}}
\end{gathered}
$$

where the energy and divergence distributions are assumed Gaussian with widths $\sigma_{\gamma}$ and $\sigma_{x^{\prime}}$. In particular, at small angles, the cone width depends on the number of wiggler periods $N_{w}$, energy spread and divergence. While the contribution from $N_{w}$ is known, the contributions from energy spread and divergence are of similar magnitude and appearance. However, at large off-axis angles, the cone width due to $N_{w}$ approaces a $\delta$ function. This permits a method for avoiding the resolution limition imposed by the on-axis width of $1 / \sqrt{N_{w}} \gamma$. At large cone angles, the contribution from energy spread also becomes increasingly negligible. Thus, divergence can extracted at large cone angles simply by measuring cone width, and simultaneous measurement of large and small cones allows divergence and energy spread to be separated and quantified.

The experimental setup is similar to that of Fig. 1, but with a narrowband interference filter and CCD camera immediately intercepting the emissions outcoupled from the beamline. The measurements were performed at the ATF at BNL [8], [23], using a train of 20 
microbunches at 150-200 pC, $48 \mathrm{MeV}, 0.5 \%$ full width energy spread and a few $\pi \mathrm{mm} \operatorname{mrad}$ emittance. The beam was sent through the MIT microwiggler [29], for which $\lambda_{w}=8.8 \mathrm{~mm}$, producing visible emissions (532 nm).

Experimental results are shown in Fig. 9. Intercepted cones for four values of beam energy, $\gamma$, near $48 \mathrm{MeV}$ are presented in Fig. 9a, showing excellent sensitivity to small changes $(0.5 \%)$ in mean beam energy. At increasingly large cone angles $(>1.02 \times 48 \mathrm{MeV})$, the cone width reached an asymptotic value of $250 \mu \mathrm{rad}$, yielding a single shot measure of the beam divergence. Fig. 9b shows that the response of small angle cones to small changes ( $0.5 \%)$ in beam energy spread could be resolved, while beam steering to the right of the wiggler center, resulting in trajectory curvature inside the wiggler, produced change to the washed out image in Fig. 9c. Marked changes in cone width $\left(\theta_{\text {cone }}\right.$ reaching $\left.1.5 \mathrm{mrad}\right)$ were observed when different slices (head, middle, tail, $>1$ ps each) of the beam passed through a selector.

\section{LASER-BASED TECHNIQUES}

The use of ultrafast lasers in combination with electron beams has presented a new vista in electron beam diagnosis: the measurement of the phase space properties of femtosecondlength electron beam slices. This type of technique will be important for the diagnosis of electron bunches of future accelerators. In Thomson scattering (TS) experiments at

the Beam Test Facility (BTF) [38] at the Advanced Light Source at Lawrence Berkeley National Laboratory, 300 fs X-ray pulses have been produced by scattering $50 \mathrm{MeV}$ electron beams against an ultrashort laser pulse in a 90 geometry [24-26] In this geometry, the radiation pulse length is determined by the limited interaction time of the ultrashort laser pulse as it traverses the tightly focussed electron bunch. Slice spot size, slice divergence and longitudinal bunchshape techniques have been developed using the Thomson scattered X-rays.

The experimental setup is shown in Fig. 10. A $50 \mathrm{MeV}, 0.2-0.4 \%$ energy spread, 10-15 
ps electron beam with $1.3 \mathrm{nC}$ total charge is focussed to $<100 \mu \mathrm{m}$ spot size, where it collides at $90^{\circ}$ with a $100 \mathrm{fs}, 40 \mathrm{~mJ}$ laser pulse with $800 \mathrm{~nm}$ central wavelength focussed to $30 \mu \mathrm{m}$, producing a $300 \mathrm{fs}$ pulse of $30 \mathrm{keV}$ X-rays. A retractable Al foil at the interaction point enabled OTR measurements to be performed at the interaction point, and compared with the Thomson scattered X-rays. The OTR radiation was either imaged with a 16 bit cooled CCD for transverse profiling of the beam spot size or sent to a streak camera with 1.5-2 ps resolution for time resolved measurements. Using the streak camera, longitudinal bunchshape and time resolved emittance (time resolved spot size or divergence) were measured (Fig. 11).

Three types of slice measurements were performed with the Thomson scattered X-rays: electron beam spot size, divergence measurement and longitudinal bunch profile. The transverse electron beam distribution for a given slice of the electron beam was obtained by scanning the laser beam transversely across the electron beam in $10 \mu \mathrm{m}$ steps and monitoring the x-ray yield on the phosphor screen. Slice divergence measurements were performed by measuring the spatial width of the X-rays and by measuring the X-ray spectrum at fixed off-axis angles. The longitudinal bunch shape was obtained by scanning laser/e beam delay.

The combination of slice measurements provided by TS and integrated measurements provided by OTR proved to be a powerful tool for studying time-correlated phase space properties of the electron beam. When the beam contains chromatic aberrations, different temporal slices of the bunch will be focused at different longitudinal locations, affecting the transverse overlap between the laser and electron beam, and the TS X-ray yield as a function of laser delay will differ from the time resolved bunchshape measurement provided by the OTR streak images. Chromatic aberrations were controlled and removed using this diagnostic: when slice and integrated bunch shape measurements were tuned to agree (Fig. 12), slice and integrated transverse profiles measured with TS and OTR agreed as well.

When the number of laser periods is large, the TS spectral flux density is proportional to the electron energy distribution [39]. TS can, for example, provide essential diagnostic information the ultrashort ( $<100 \mathrm{fs}$ ) electron bunches produced via laser wakefield acceleration. Broad electron distributions $\left(f_{g}(\gamma)=0.24 e^{-0.3 \gamma}[40]\right)$ characterize the Self Modulated 
Wakefield Accelerator [41,42] while proposed configurations such as Colliding Pulse Injector [43-45] seek to reduce the energy spread to $1 \%$. TS spectral flux densities plotted for these two cases (Fig. 13) exhibit a strong energy distribution signature.

\section{CONCLUSION}

Practical implementations of radiation-based beam diagnosis, which are well suited for ultrashort electron bunches have been discussed and cover an array of properties of $30 \mathrm{MeV}-30$ $\mathrm{GeV}$ electron beams. The development of fs electron bunches is under vigorous experimental and theoretical pursuit, with a movement toward potential applications in ultrafast science. The special properties of electron beam radiation mechanisms in synergy with ultrashort laser techniques can provide exciting new opportunities for diagnosis, microprobing and control of beams on a fs timescale.

\section{ACKNOWLEDGMENTS}

We express our thanks to J. Wurtele, M. Zolotorev, R. Schoenlein, C.V. Shank, A. Chin, T. Glover, P. Volfbeyn, P. Balling, K.J. Kim, S. Chattopadhyay at LBNL, R. Siemann (SLAC), C. Joshi (UCLA), T. Katsouleas (USC) and the members of the E157 Collaboration,

I. Ben-Zvi and the Accelerator Test Facility at BNL, R. Stoner and G. Bekefi's group at MIT and the members of the l'OASIS group at LBNL for their contributions to this work. This work was funded by the Department of Energy, Grant DE-AC-03-76SF0098. 


\section{REFERENCES}

[1] in Advanced Accelerator Concepts, Ninth Workshop, A.I.P. Conf. Proc., edited by P. L. Colestock and S. Kelley (American Institute of Physics, Melville, NY, 2001), Vol. 569.

[2] Y. Liu, X. J. Wang, D. B. Cline et al., Phys. Rev. Lett. 80, 4418 (1998).

[3] E. Esarey, P. Sprangle, J. Krall, and A. Ting, IEEE Trans. Plasma Sci. 24, 252 (1996).

[4] M. D. Perry and G. Mourou, Science 264, 917 (1994).

[5] K.-J. Kim, in Physics of Particle Accelerators, AIP Conf. Proc. (American Institute of Physics, N.Y., 1989), Vol. 184, pp. 565-632.

[6] R. Barbini, F. Ciocci, G. Dattoli, and L. Giannessi, Riv. Nuovo Cimento 13 ser.3, 1 (1990).

[7] A. H. Lumpkin, Nucl. Instr. \& Meth. A 296, 134 (1990).

[8] P. Catravas, Ph.D. thesis, Massachusetts Institute of Technology, 1998.

[9] M. S. Zolotorev and G. V. Stupakov, SLAC-PUB-7132 (1996).

[10] M. S. Zolotorev and G. V. Stupakov, in Proceedings of the 1997 Particle Accelerator Conference, edited by M. Comyn, M. K. Craddock, M. Reiser, and J. Thomson (The Institute of Electrical and Electronics Engineers, Inc., Piscataway, NJ, 1998), Vol. 2, pp. $2180-2$.

[11] P. Catravas, W. P. Leemans, J. S. Wurtele et al., Phys. Rev. Lett. 82, 5261 (1999).

[12] V. Sajaev, in Proceedings of the Seventh European Particle Accelerator Conference, edited by J. Poole and C. Petit-Jean-Genaz (Institute of Physics, London, UK, 2000), pp. $1806-8$.

[13] L. Wartski, S. Roland, J. Lasalle, M. Bolore, and G. Filippi, J. Appl. Phys. 46, 3644 (1975). 
[14] D. W. Rule, Nucl. Instr. \& Meth. B 24-25, 901 (1987).

[15] D. W. Rule, R. B. Fiorito, A. H. Lumpkin, R. B. Feldman, and B. E. Carlsten, Nucl. Instr. \& Meth. A 296, 739 (1990).

[16] A. H. Lumpkin, Nucl. Instr. \& Meth. A 393, 170 (1997).

[17] A. H. Lumpkin, R. B. Fiorito, D. W. Rule, D. H. Dowell, W. C. Sellyey, and A. R. Lowrey, Nucl. Instr. \& Meth. A 296, 150 (1990).

[18] A. H. Lumpkin and C. E. Eyberger, Beam Instrumentation : Proceedings of the Seventh Workshop : Argonne, Illinois, May, 1996 (AIP Press, Woodbury, New York, 1997).

[19] W. P. Leemans, in Advanced Accelerator Concepts Workshop. AIP Conf. Proc., edited by S. Chattopadhyay (American Institute of Physics, Woodbury, NY, 1997), Vol. 398, pp. 23-39.

[20] P. Catravas, W. P. Leemans, E. Esarey et al., in Proceedings of the 1999 Particle Accelerator Conference, edited by A. Luccio and W. MacKay (The Institute of Electrical and Electronics Engineers, Inc., Piscataway, NJ, 1999), Vol. 3, pp. 2111-13.

[21] M. J. Hogan, R. Assmann, F. J. Decker et al., Phys. Plasmas 7, 2241 (2000).

[22] P. Catravas, S. Chattopadhyay, E. Esarey et al., Phys. Rev. E 64, 046502 (2001).

[23] X. Qiu, P. Catravas, M. Babzien et al., Nucl. Instr. \& Meth. A 393, 484 (1997).

[24] W. P. Leemans, R. W. Schoenlein, P. Volfbeyn et al., Phys. Rev. Lett. 77, 4182 (1996).

[25] W. P. Leemans, R. W. Schoenlein, P. Volfbeyn et al., IEEE J. Quantum Electron. 33, 1925 (1997).

[26] R. W. Schoenlein, W. P. Leemans, A. H. Chin et al., Science 274, 236 (1996).

[27] M. S. Gupta, Proc. IEEE 63, 996 (1975).

[28] X. J. Wang, M. Babzien, K. Batchelor et al., Nucl. Instr. \& Meth. A 375, 82 (1996). 
[29] R. Stoner and G. Bekefi, IEEE J. Quantum Electron. 31, 1158 (1995).

[30] V. L. Ginzburg and I. M. Frank, Sov. Phys. JETP 16, 15 (1946).

[31] L. C. L. Yuan, C. L. Wang, H. Uto, and S. Prunster, Phys. Rev. Lett. 25, 1513 (1970).

[32] R. B. Fiorito, D. W. Rule, A. H. Lumpkin, R. L. Tokar, D. H. Dowell, W. C. Sellyey, and A. R. Lowrey, in Proceedings of the 1991 Particle Accelerator Conference (The Institute of Electrical and Electronics Engineers, Inc., N.Y., 1991), Vol. 2, pp. 1204-6.

[33] W. K. H. Panofsky and M. Phillips, Classical Electricity and Magnetism, AddisonWesley Series in Physics, 2d ed. (Addison-Wesley Pub. Co., Reading, MA, 1962).

[34] J. D. Jackson, Classical Electrodynamics (John Wiley \& Sons, Inc., N.Y., 1998).

[35] C. R. Vidal and J. Cooper, J. Appl. Phys. 40, 3370 (1969).

[36] A.I.P. Handbook, edited by D. E. Gray (McGraw Hill, New York, 1972).

[37] C. E. Clayton, B. E. Blue, E. S. Dodd et al., submitted to Phys. Rev. Lett. .

[38] W. Leemans, G. Behrsing, K. J. Kim, J. Krupaick, C. Matuk, F. Selph, and S. Chattopadhyay, in Proceedings of the 1993 Particle Accelerator Conference, edited by S. T. Corneliussen (The Institute of Electrical and Electronics Engineers, Inc., NY, 1993), Vol. 1, pp. 83-5.

[39] P. Catravas, E. Esarey, and W. P. Leemans, Meas. Sci. Technol. 12, 1828 (2001).

[40] W. P. Leemans, D. Rodgers, P. E. Catravas et al., Phys. Plasmas 8, 2510 (2001).

[41] E. Esarey, J. Krall, and P. Sprangle, Phys. Rev. Lett. 72, 2887 (1994).

[42] W. B. Mori, C. D. Decker, D. E. Hinkel, and T. Katsouleas, Phys. Rev. Lett. 72, 1482 (1994).

[43] E. Esarey, R. F. Hubbard, W. P. Leemans, A. Ting, and P. Sprangle, Phys. Rev. Lett. 79, 2682 (1997). 
[44] C. B. Schroeder, P. B. Lee, J. S. Wurtele, E. Esarey, and W. P. Leemans, Phys. Rev. E 59, 6037 (1999).

[45] E. Esarey, C. B. Schroeder, W. P. Leemans, and B. Hafizi, Phys. Plasmas 6, 2262 (1999). 


\section{FIGURES}

FIG. 1. Fluctuational interferometry experimental setup (after ref. [11]).

FIG. 2. Measured single shot spontaneous emission spectra for 1.5 and 4.5 ps bunchlengths are compared in a) and b) and show a reduction in the characteristic spike width at the longer bunchlength. Numerically generated spectra in c) and d) for the experimental conditions of a) and b), respectively, reproduce the salient features of the data - the characteristic spike width and level of modulation (after ref. [11]).

FIG. 3. a) The measured intensity distribution for spectra at the shortest (1.5 ps) and longest bunchlengths (4.5 ps) are well fit by the gamma distribution (solid line, Eqn. 2) evaluated at $\mathrm{k}=2.0$ and 3.6, respectively. b) Average spectral intensity correlation (Eqn. 5) for the shortest and longest bunchlengths are shown, and permit the correlation length, $1 / \tau_{b}$, and the number of transverse modes to be separately quantified through fitting with Eqn. 6 (solid line) (after ref. $[11])$.

FIG. 4. Experimental setup - OTR diagnosis at $30 \mathrm{GeV}$ (after ref. [20]).

FIG. 5. a) Electron beam size (50 $\mu \mathrm{m}$ minimum) extracted using OTR imaging (shown to right) for a quadrupole scan was in agreement with independent measurements. b)Measurements of the 2-foil OTR angular distribution for high and low beam divergence are shown along with horizontal lineouts (after ref. [20]).

FIG. 6. Cerenkov radiation experimental setup (after ref. [22]).

FIG. 7. Cerenkov radiation in images of the downstream OTR foil (right) were used to estimate neutral density as a function of oven temperature and were compared with known values, shown with solid line (after ref. [22]). 
FIG. 8. Plasma density extracted from the Cerenkov cone radius (left) is compared with plasma density extracted from betatron oscillations(right) monitored in images similar to those shown in Fig. 7 (after ref. [22]).

FIG. 9. Narrow bandwidth cones $(532 \mathrm{~nm}, 1 \mathrm{~nm} \mathrm{BW})$ generated with a 70 period microwiggler respond sensitively to a) beam energy, b) energy spread and c) steering. Beam divergence was extracted from the measurements shown in a) (after ref [8].)

FIG. 10. Schematic of Thomson scattering experiment with $90^{\circ}$ scattering geometry (after ref. $[24])$.

FIG. 11. Time resolved divergence of a $50 \mathrm{MeV}$ electron beam was measured with a streak camera (left) imaging the OTR radiation angular distribution. The lineout (right) shows the effect of divergence (after ref. [19]).

FIG. 12. Bunchshape measured with Thomson scattered X-rays and time resolved OTR differ (left) in the presence of chromatic aberrations and agree (right) when the aberrations are removed (after ref. [24]).

FIG. 13. Thomson scattering spectral flux density are contrasted for the energy distributions of two laser wakefield accelerator configurations, self-modulated laser wakefield (left) and colliding pulse configuration (right) (after ref. [39]). 


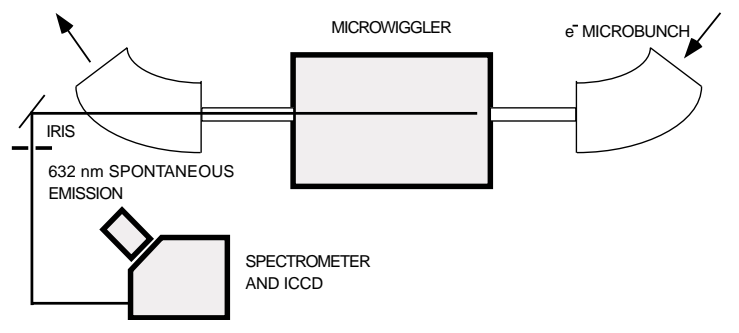



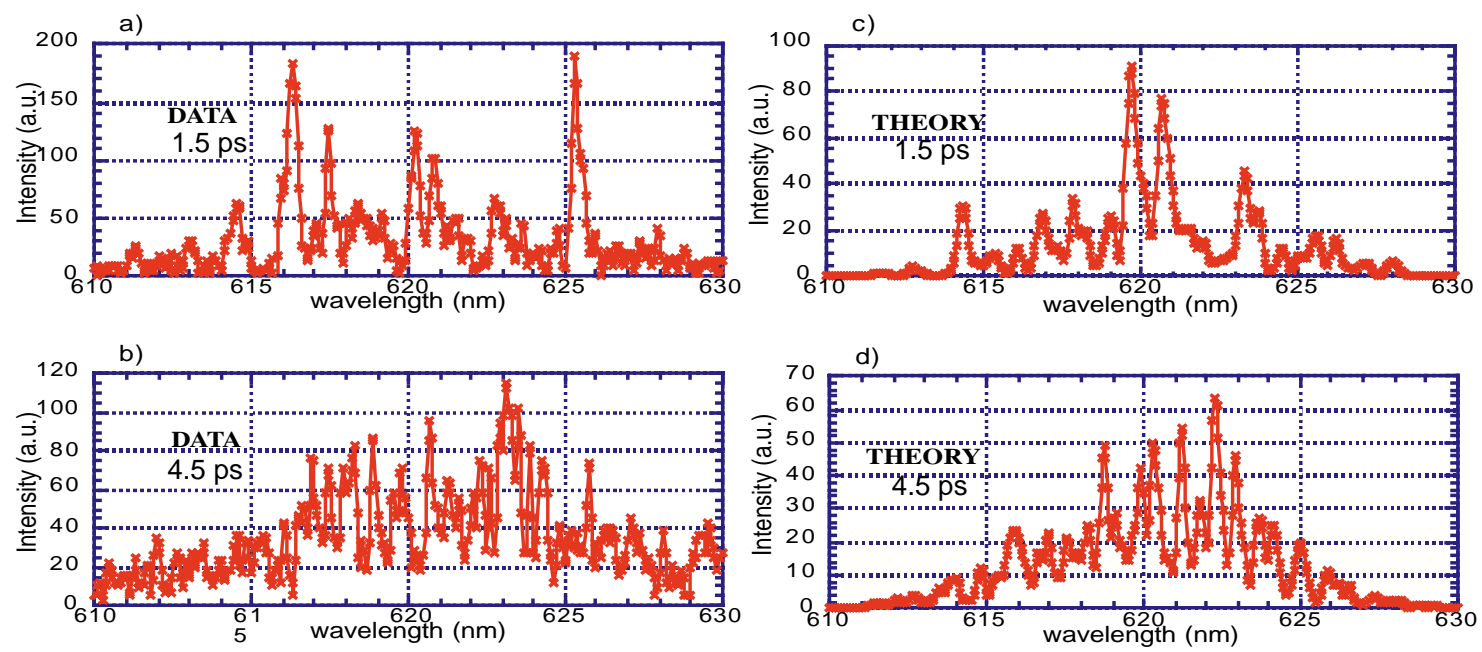

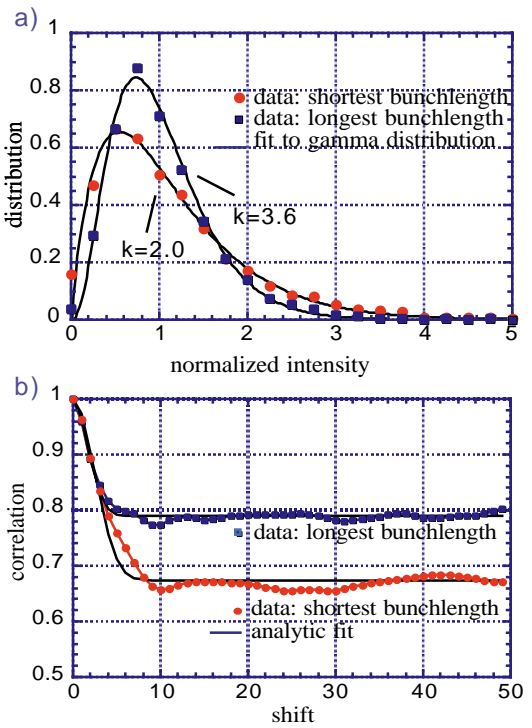


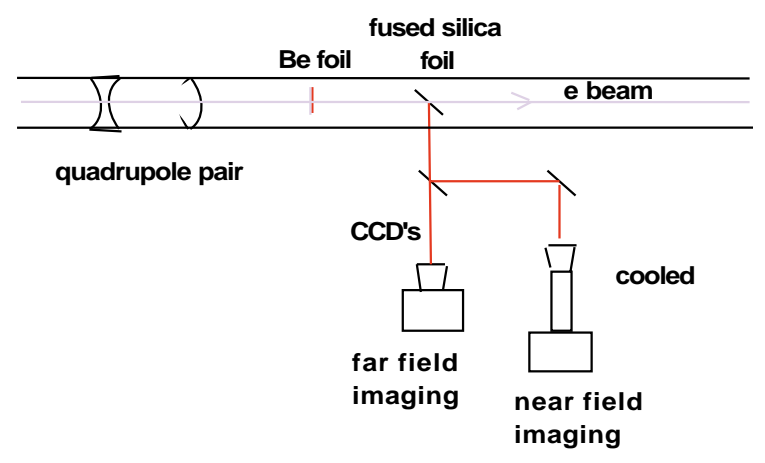



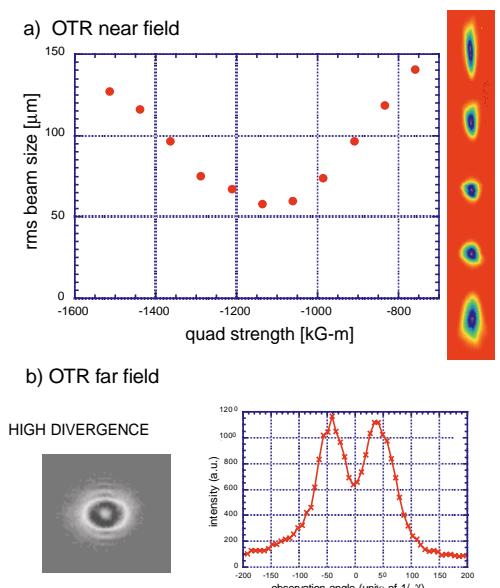

LOW DIVERGENCE

(O)

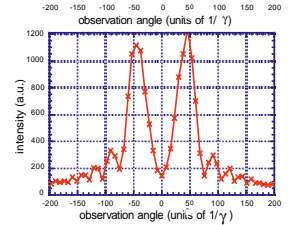




OTR foil




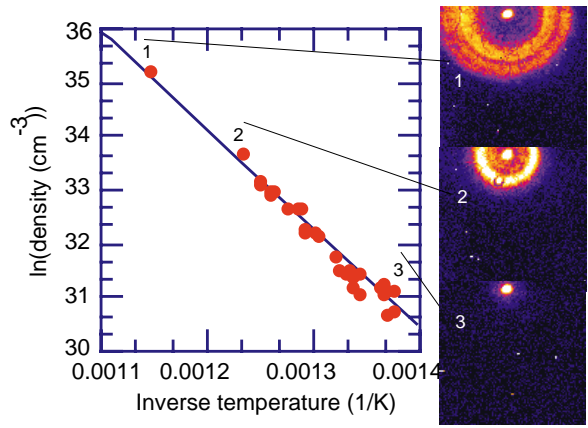



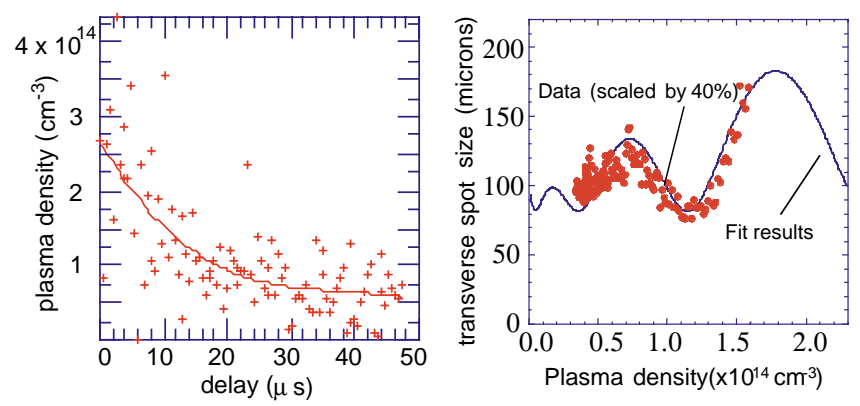


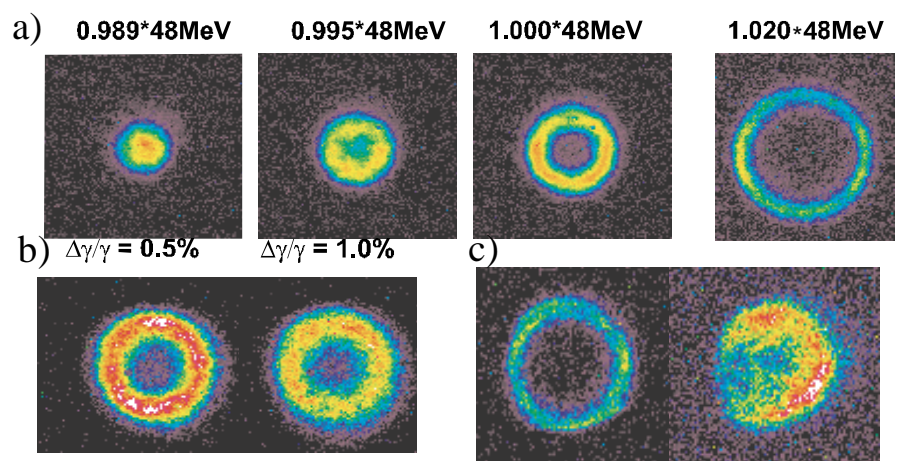




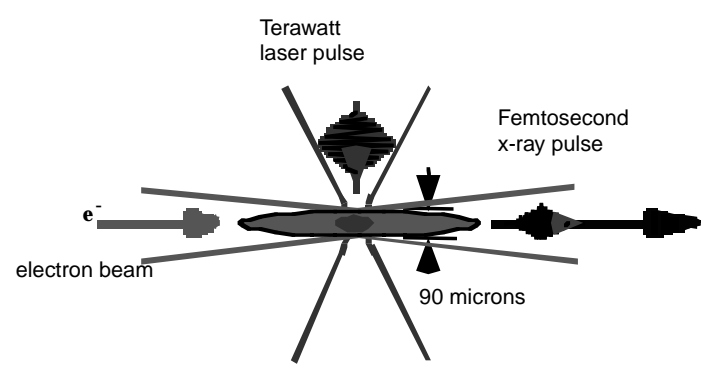


(a)

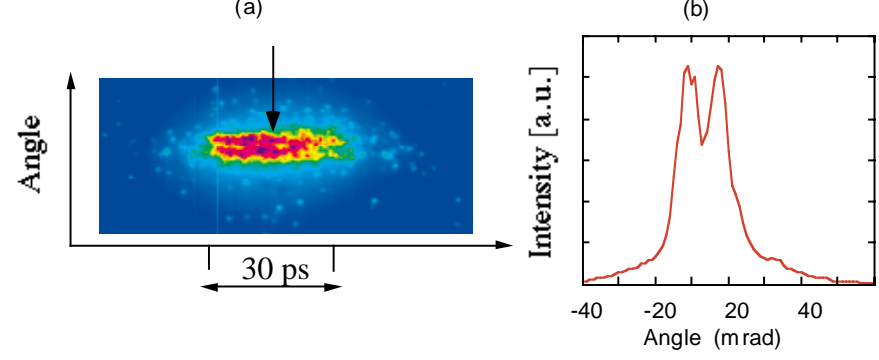




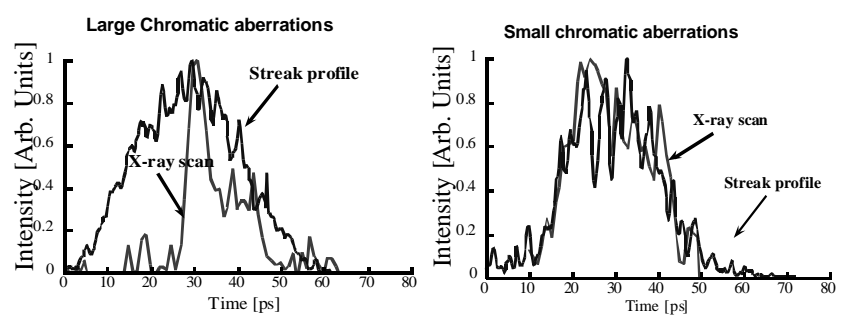




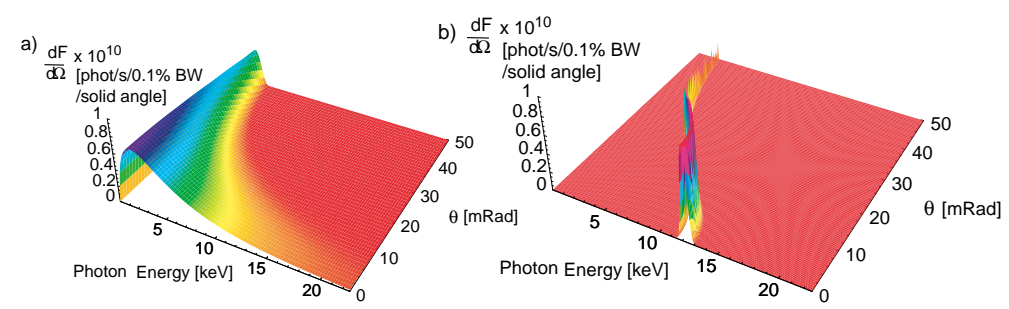

